\title{
Inteligencias Múltiples en el trabajo docente y su relación con la Teoría del Desarrollo Cognitivo de Piaget
}

\section{Multiple Intelligences in teaching work and its relationship with Piaget's Theory of Cognitive Development}

\author{
Zaira Ramírez Apud López ${ }^{1 *}$ y Tammara Ramírez Apud López ${ }^{1}$ \\ ${ }^{1}$ Benemérita Universidad Autónoma de Puebla \\ *zaira.ramirezap@ correo.buap.mx
}

DOI: https://doi.org/10.26871/killkana_social.v2i2.299

\begin{abstract}
Resumen
En este artículo se hace una revisión teórica sobre el concepto de Inteligencia, aterrizando en las Inteligencias Múltiples de Gardner (1997) y su asociación con la Teoría del Desarrollo Genético de Piaget (1968), con la finalidad de establecer una propuesta que relaciona las inteligencias de acuerdo con las características de Piaget y que estas, a su vez respondan a las inteligencias que deben tener los docentes que se encuentran desempeñándose en el Nivel Inicial y Educación General Básica. En este documento se relacionan las Inteligencias que requieren los docentes dependiendo del nivel educativo en el que participan, donde las inteligencias se clasificaron de acuerdo con las características que Piaget propone en su Teoría del Desarrollo Cognitivo, con base en las edades de los niños que cursan la Educación Inicial y la Básica. La propuesta va encaminada a los docentes que se dedican a trabajar con niños de Educación Inicial y Educación Básica. El análisis busca asociar las inteligencias que pueden apoyar a los docentes dependiendo las características cognitivas de los niños, planteadas por Piaget en su Teoría de Desarrollo Genético, y asociadas a las Inteligencias Múltiples de Gardner.
\end{abstract}

Palabras clave: Inteligencias Múltiples, Estadios de Piaget, Docentes en Educación Inicial y Básica.

\begin{abstract}
In this article a theoretical revision is made about the concept of Intelligence, landing in the Multiple Intelligences of Gardner (1997) and its association with the Theory of the Genetic Development of Piaget (1968), with the purpose of establishing a proposal that relates the intelligences according to the characteristics of Piaget and that these, in turn, respond to the intelligences that must have the teachers who are working in the Initial Level and Basic General Education. In this document are related the Intelligences that the teachers require depending on the educational level in which they participate, where the intelligences were classified according to the characteristics that Piaget proposes in his Theory of Cognitive Development, based on the ages of the children that attend Initial and Basic Education. The proposal is aimed at teachers who work with children from Initial Education and Basic Education. The analysis seeks to associate the intelligences that can support the teachers depending on the cognitive characteristics of the children, raised by Piaget in his Theory of Genetic Development, and associated with the Multiple Intelligences of Gardner.
\end{abstract}

Key words: Multiples Intelligence, Piaget's cognitive stages, teachers of Early Childhood and Basic General Education.

\section{Introducción}

En este estudio se presenta una breve revisión sobre los principales teóricos del concepto de inteligencia, los cuales son un antecedente a la propuesta de las inteligencias múltiples realizada por Howard Gardner (1983).

Las inteligencias múltiples llegan a revolucionar la concepción tradicional que se tenía de la inteligencia y uno de los aspectos importantes es que esta se puede desarrollar a partir de lo genético, pero incorporando lo social. Se deja atrás la concepción de inteligencia como un factor medible
(CI) y que recae solamente en habilidades como la lógica matemática y la lingüística.

A partir de la propuesta de Gardner (1983), se puede identificar el tipo de inteligencia de una persona, lo que permite conocer el ambiente donde tendría un mejor aprendizaje.

Tomando en cuenta lo anterior, para este estudio se presenta una propuesta que logre integrar la Teoría Genética de los estadios de desarrollo de Piaget (1968), con las Inteligencias Múltiples de Gardner (1983), dependiendo 
el nivel educativo de los niños con los que trabajan los docentes.

\section{Antecedentes}

Se debe comenzar por definir qué se entiende por Inteligencia y cuál ha sido la evolución del concepto hasta integrarse con el de Inteligencias Múltiples de Gardner(1983).

\subsection{Concepto de inteligencia}

Según la Real Academia de la Lengua (RAL), proviene del latín intelligentia, y se refiere a la capacidad de conocer, entender, resolver problemas, así como también, habilidad, destreza y experiencia (Real Academia Española, 2001).

Desde la definición se plantea un concepto amplio de la inteligencia en donde la persona que sobresale debe tener características integradoras que le permitan desenvolverse en cualquier ambiente, ya sea académico o social.

Su estudio ha tenido una larga historia para buscar su conceptualización, por una parte, se tienen las Teorías Legas, donde la inteligencia forma parte del sentido común que las personas utilizan para explicar y predecir fenómenos de su entorno (Ramírez y Levy, 2009).

En este concepto se da importancia a un aspecto lógico, en el que por sentido común la persona tiene la suficiente información para anticiparse a los sucesos que se le presentan.

Por otra parte está el Enfoque Psicométrico Común, en el que se define Inteligencia a partir de pruebas que permitan medirla, a partir de este enfoque surgen las "pruebas de CI" (Gould, 1981, en Gardner 1993, p.6), mismas que llegaron a implementarse en el sistema educativo, para empezar a estandarizar la inteligencia.

\subsection{Coeficiente intelectual (CI)}

Otro teórico que continuó con la estandarización de la Inteligencia fue Alfred Binet (1857-1911), quien lleva a cabo los primeros intentos por medirla, como una respuesta a las necesidades de las escuelas francesas del nivel básico y cuya finalidad era seleccionar a los estudiantes que contaran con las habilidades para ingresar y asegurar un buen rendimiento académico (Ardila, 2010, p.98).

De acuerdo con lo anterior, se puede observar que el sistema educativo empieza a retomar los estudios que impliquen medir la inteligencia para conocer si una persona es sobresaliente o pueden identificar a quienes tienen un nivel normal o por debajo de lo normal. Estas pruebas todavía se siguen aplicando, aunque es necesario que sean reaplicadas en diferentes momentos porque el ser humano responde de acuerdo al ambiente o situación en la que se encuentre.

El sistema escolar estaba interesado en identificar a los mejores estudiantes para garantizar el rendimiento académico sin embargo, esta premisa no pierde vigencia en la actualidad varias instituciones consideran este factor importante, en ocasiones invierten en aplicar pruebas para medir la inteligencia con el fin de clasificar a las personas de acuerdo a su CI, que pueden ser bajo, promedio y alto, a partir de los rangos obtenidos con estos instrumentos.

Para Binet, citado por Ardila (2010, p.98), se define la inteligencia como aquellas cualidades formales como la memoria, la percepción, la atención y el intelecto. Es a partir de estas que desarrolla sus estudios para medir y clasificar el desempeño del pensamiento humano.

Con base en los estudios de Binet, se plantea el concepto de Coeficiente Intelectual (CI), propuesto por William Stern (1871- 1938), el cual lo define como una capacidad que puede ser medible y resalta las diferencias que existen de un individuo a otro (Stern, 2018, p.1).

El Coeficiente Intelectual (CI) representa una forma de conocer el nivel de inteligencia de los individuos para poder identificar a aquellos que pueden ser considerados como sobresalientes. Tomando en cuenta que con este coeficiente se nace y a partir de ser identificado, se puede seguir aumentando el nivel de inteligencia con los estímulos adecuados.

\subsection{Inteligencia a partir de la solución de problemas}

En las Teorías de Pluralización, los estudios se encuentran basados en los trabajos de Charles Spearman (1927) y Lewis Terman (1975), quienes veían la Inteligencia como la capacidad única de formar conceptos y resolver problemas. Finalmente, estudiosos como Thurston y Guilford, comienzan a plantear la existencia de varios factores o componentes de la Inteligencia (Gardner, 1983, p.6).

En la propuesta para contextualizar la Inteligencia, están los estudios realizados por Berg y Stenberg en (Pérez y Medrano, 2013), quienes reconocen que adicionalmente a la capacidad de adaptación, la inteligencia explica las diferencias que observamos entre las personas cuando estas resuelven problemas, razonan y/o toman decisiones.

Tabla 1. Las ocho inteligencias múltiples de Gardner y sus características

\begin{tabular}{|c|l|}
\hline $\begin{array}{c}\text { Tipos de } \\
\text { Inteligencias }\end{array}$ & Características \\
\hline $\begin{array}{c}\text { Verbal / } \\
\text { Lingǘstica }\end{array}$ & $\begin{array}{l}\text { Es la capacidad de emplear de manera eficaz las } \\
\text { palabras, manipulando la estructura o sintaxis del } \\
\text { lenguaje, la fonética, la semántica, y sus dimensio- } \\
\text { nes prácticas. } \\
\text { Está en los niños a los que les encanta redactar } \\
\text { historias, leer, jugar con rimas, trabalenguas y en } \\
\text { los que aprenden con facilidad otros idiomas. }\end{array}$ \\
\hline Lógica / & $\begin{array}{l}\text { Es la capacidad de manejar números, relaciones y } \\
\text { patrones lógicos de manera eficaz, así como otras } \\
\text { funciones y abstracciones de este tipo. }\end{array}$ \\
$\begin{array}{l}\text { Los niños que la han desarrollado analizan con } \\
\text { facilidad planteamientos y problemas. Se acercan a } \\
\text { los cálculos numéricos, estadísticas y presupuestos } \\
\text { con entusiasmo. }\end{array}$ \\
\hline Visual / \\
Espacial & $\begin{array}{l}\text { Es la habilidad de apreciar con certeza la imagen } \\
\text { visual y espacial, de representarse gráficamente las } \\
\text { ideas, y de sensibilizar el color, la línea, la forma, } \\
\text { la figura, el espacio y sus interrelaciones. }\end{array}$ \\
\hline
\end{tabular}




\begin{tabular}{|c|c|}
\hline $\begin{array}{c}\text { Tipos de } \\
\text { Inteligencias }\end{array}$ & Características \\
\hline & $\begin{array}{l}\text { Está en los niños que estudian mejor con gráficos, } \\
\text { esquemas, cuadros. Les gusta hacer mapas concep- } \\
\text { tuales y mentales. Entienden muy bien planos y } \\
\text { croquis. }\end{array}$ \\
\hline $\begin{array}{l}\text { Musical / } \\
\text { Rítmica }\end{array}$ & $\begin{array}{l}\text { Es la capacidad de percibir, distinguir, transformar } \\
\text { y expresar el ritmo, timbre y tono de los sonidos } \\
\text { musicales. }\end{array}$ \\
\hline & $\begin{array}{l}\text { Los niños que la evidencian se sienten atraídos por } \\
\text { los sonidos de la naturaleza y por todo tipo de } \\
\text { melodías. Disfrutan siguiendo el compás con el pie, } \\
\text { golpeando o sacudiendo algún objeto rítmicamen- } \\
\text { te. }\end{array}$ \\
\hline $\begin{array}{l}\text { Corporal / } \\
\text { Kinestésica }\end{array}$ & $\begin{array}{l}\text { Es la habilidad para usar el propio cuerpo para } \\
\text { expresar ideas y sentimientos, y sus particularida- } \\
\text { des de coordinación, equilibrio, destreza, fuerza, } \\
\text { flexibilidad y velocidad, así como propioceptivas y } \\
\text { táctiles. } \\
\text { Se la aprecia en los niños que se destacan en acti- } \\
\text { vidades deportivas, danza, expresión corporal y/o } \\
\text { en trabajos de construcciones utilizando diversos } \\
\text { materiales concretos. También en aquellos que son } \\
\text { hábiles en la ejecución de instrumentos. }\end{array}$ \\
\hline Interpersonal & $\begin{array}{l}\text { Es la posibilidad de distinguir y percibir los estados } \\
\text { emocionales y signos interpersonales de los demás, } \\
\text { y responder de manera efectiva a dichas acciones } \\
\text { de forma práctica. } \\
\text { La tienen los niños que disfrutan trabajando en } \\
\text { grupo, que son convincentes en sus negociaciones } \\
\text { con pares y mayores, que entienden al compañero. }\end{array}$ \\
\hline Intrapersonal & $\begin{array}{l}\text { Es la habilidad de auto introspección, y de ac- } \\
\text { tuar consecuentemente sobre la base de este co- } \\
\text { nocimiento, de tener una autoimagen acertada, y } \\
\text { capacidad de autodisciplina, comprensión y amor } \\
\text { propio. } \\
\text { La evidencian los niños que son reflexivos, de } \\
\text { razonamiento acertado y suelen ser consejeros de } \\
\text { sus pares. }\end{array}$ \\
\hline Naturalista & $\begin{array}{l}\text { Es la capacidad de distinguir, clasificar y utilizar } \\
\text { elementos del medio ambiente, objetos, animales } \\
\text { o plantas. Tanto del ambiente urbano como subur- } \\
\text { bano o rural. Incluye las habilidades de observa- } \\
\text { ción, experimentación, reflexión y cuestionamiento } \\
\text { de nuestro entorno. } \\
\text { Se da en los niños que aman los animales, las } \\
\text { plantas; que reconocen y les gusta investigar ca- } \\
\text { racterísticas del mundo natural y del hecho por el } \\
\text { hombre. }\end{array}$ \\
\hline
\end{tabular}

Fuente: Gardner (1983 en González, 2007).

Desde la perspectiva de las IM, se reconoce que lo más importante no es saber el CI de las personas, sino conocer qué tipos de inteligencia son predominantes y cuáles están menos desarrolladas (Ander-Egg, 2006, p.4).

De acuerdo con lo anterior, al conocer las inteligencias se puede entender la forma en que se interactúa y en que se aprende. Se toman decisiones y se resuelven problemas dependiendo las inteligencias que tenemos. Al mismo tiempo, se pueden desarrollar las que se obtuvieron como poco desarrolladas para alcanzar otras inteligencias.

\subsection{El docente y sus tipos de inteligencia}

Es relevante conocer como docentes la relación que existe entre el perfil de inteligencias que se tiene con las características de los alumnos con los que se trabaja en el Sistema Educativo.

El docente debe identificar su propio perfil para tenerlo presente en el aprendizaje que promueve en el aula. Dependiendo de sus inteligencias implementará estrategias que le permitan interactuar y facilitar el aprendizaje en los niños que se encuentran en el sistema educativo.
Un docente que trabaja con niños del nivel de Educación Inicial deberá tener desarrolladas inteligencias diferentes al docente de Educación General Básica. Los primeros requieren ser dinámicos y propiciar un aprendizaje a través de los sentidos y los segundos deben propiciar el razonamiento, la lógica, entre otros.

Para proponer una relación entre las Inteligencias Múltiples del docente y las necesidades de los niños en el sistema educativo, se consideró que para el nivel de Educación Inicial y Básica, tienen características que les facilitan el aprendizaje con base en su edad. Por lo anterior se retoma la Teoría Constructivista Genética que realiza Piaget (1968), donde identifica cómo se desarrolla el intelecto en el ser humano desde la infancia y propone estadios de desarrollo del ser humano.

A partir esta propuesta, se podrá observar cómo el perfil de inteligencias puede tener características que requiere el docente, para dar atención a niños de nivel Inicial o Básico.

\subsection{Constructivismo Genético de Jean Piaget}

Jean William Fritz Piaget (1896-1980) fue un epistemólogo, psicólogo y biólogo que acuña la Teoría del Desarrollo del Niño, basada en el "constructivismo genético", el cual plantea los estadios de desarrollo de la inteligencia y de los conocimientos científicos (Munari, 1994, p.6).

Se puede observar que Piaget también maneja el término de inteligencia y cómo se desarrolla a partir de la madurez mental del ser humano, donde influye la edad y los estímulos del ambiente, es decir la realidad en la que interactúa.

Piaget plantea que gracias a la disposición interna característica del organismo (inteligencia), el ser humano tiene una manera única y específica de interactuar con el medio ambiente para organizar estructuralmente el conocimiento a partir de la acción (Wallon, 1980en Cárdenas, 2011).

Resalta el planteamiento de que la inteligencia es parte de los factores fisiológicos del individuo y es a partir de la interacción con el medio ambiente que va a ir desarrollando estructuras cognitivas que se van fortaleciendo, dependiendo de los estímulos que se reciban en el contexto.

A continuación se presentan los estadios de desarrollo, planteados por Piaget (1968 en Saldarriaga, Bravo y Loor, 2016) que caracterizan al niño de acuerdo con su edad y la fase en la que se encuentran.

Tabla 2. Estadios de desarrollo de Jean Piaget (1968).

\begin{tabular}{|c|l|}
\hline Estadio & Características \\
\hline & $\begin{array}{l}\text { Caracterizado por el desarrollo de los reflejos, la } \\
\text { construcción del conocimiento comienza con el }\end{array}$ \\
Etapa & ejercicio de los reflejos innatos, que luego permiten \\
el desarrollo de los esquemas por el ejercicio y la \\
sensoriomotriz & coordinación hasta llegar al descubrimiento de pro- \\
& cesamientos mentales. Se presenta la adquisición \\
& de la función simbólica o capacidad de representar \\
& el mundo externo por medio de símbolos. \\
\hline
\end{tabular}




\begin{tabular}{|c|l|}
\hline Estadio & Características \\
\hline $\begin{array}{c}\text { Etapa } \\
\text { preoperatoria } \\
\text { (2 a 7 años) }\end{array}$ & $\begin{array}{l}\text { Surge la función simbólica donde el niño piensa so- } \\
\text { bre hechos u objetos no perceptibles. La inteligen- } \\
\text { cia o razonamiento es intuitivo y no posee lógica. } \\
\text { Utiliza esquemas representativos como el lenguaje, } \\
\text { juego simbólico y el dibujo. Suelen atribuir vida } \\
\text { y características subjetivas a objetos inanimados, } \\
\text { pues comprenden la realidad a parte de los esque- } \\
\text { mas mentales que poseen. }\end{array}$ \\
\hline $\begin{array}{c}\text { Etapa de } \\
\text { operaciones } \\
\text { concretas (7 }- \\
12 \text { años })\end{array}$ & $\begin{array}{l}\text { guiar por las apariencias perceptivas. Su pensa- } \\
\text { miento es concreto y puede clasificar, seriar, en- } \\
\text { tiende la noción de número. Establece relaciones } \\
\text { cooperativas, toma en cuenta el punto de vista de } \\
\text { los demás. Empieza a construir una moral. }\end{array}$ \\
\hline $\begin{array}{c}\text { Etapa de } \\
\text { operaciones } \\
\text { formales (12 } \\
\text { años en } \\
\text { adelante) }\end{array}$ & $\begin{array}{l}\text { Desarrolla la inteligencia formal. El pensamiento } \\
\text { es reversible, interno y organizado. Comprende el } \\
\text { conocimiento científico, elabora hipótesis y razona } \\
\text { sin tener presentes los objetos. Esa estructura del } \\
\text { pensamiento es parte de la pre-adolescencia y es } \\
\text { cuando combina objetos sistemáticamente. }\end{array}$ \\
\hline
\end{tabular}

Fuente: Elaboración propia a partir de la información de Saldarriaga, Bravo y Loor (2016) y de Rosas y Sebastián (2008)

De acuerdo con Saldarriaga, Bravo y Loor (2016, p.133), de la clasificación que hace Piaget a partir de los Estadios del Desarrollo Cognoscitivo se observa que la inteligencia es una cualidad inherente del hombre y que los seres humanos son inteligentes en todas las edades solo que de manera distinta, constituyendo esta inteligencia una herramienta esencial para su adaptación al medio.

Como se puede observar esta propuesta de inteligencia es muy cercana a la realizada por Gardner (1997), donde también presenta que todo ser humano es inteligente, pero es resultado del contexto. Por lo anterior, existe congruencia entre los planteamientos de Piaget y Gardner.

\section{Propuesta}

La propuesta va encaminada a los docentes que se dedican a trabajar con niños de Educación Inicial y Educación Básica. El análisis busca asociar las inteligencias que pueden apoyar a los docentes dependiendo las características cognitivas de los niños, planteadas por Piaget en su Teoría de Desarrollo Genético, y asociadas a las Inteligencias Múltiples de Gardner. A continuación, se presenta su planteamiento desde el nivel educativo en el que se desempeña el docente.

\subsection{IM en docentes de Educación Inicial}

Considerando la edad de los niños que se encuentran en Educación Inicial (de 0 a 5 años), y de acuerdo con la Teoría de Piaget (1968), están en el Estadio Sensorio Motriz y el preoperatorio. En este estadio aprenden a través de los sentidos y el movimiento, lo cual habla del desarrollo de habilidades corporales y motrices que ayudan al manejo de herramientas o de ciertas emociones como la inteligencia lo describe (Regader, 2018).

Teniendo en cuenta lo anterior, las características que tiene los niños, del Nivel Inicial, de acuerdo con el desarrollo cognitivo que presentan, se considera que las Inteligencias que deberían desarrollar los docentes que se dediquen a este nivel educativo serían:

\section{- Corporal / Kinestésica}

- Visual / Espacial

- Lingüística / Verbal

- Musical

- Interpersonal

Debido a que cumplen con las características que propone Piaget en las etapas del desarrollo cognitivo de los niños que cursan educación inicial y además considera las características que describe Gardner (1997), para estas inteligencias.

Brevemente se explica por qué se seleccionaron esas inteligencias para los docentes que se encuentran en el Nivel de Inicial, a partir de la Etapa Sensorio Motriz. La inteligencia Corporal / Kinestésica, porque el niño aprende en movimiento. Visual / Espacial, porque los estímulos que reciba del entorno, como colores llamativos o material didáctico, son importantes para desarrollar la inteligencia. Lingüística / Verbal, es fundamental porque el niño amplía su vocabulario mucho más que en otras etapas de desarro1lo. Musical/Rítmica, donde a parte de aprender a través del movimiento, la música implica el manejo de emociones. Interpersonal, donde el niño tiene la capacidad de aprender con los otros.

Si el docente tiene este cuadro de inteligencias puede facilitar el desarrollo cognitivo del niño del Nivel de Educación Inicial porque estaría cubriendo sus características de acuerdo con los estadios de Piaget.

\subsection{IM en docentes de Educación Básica}

Los niños que se encuentran en el Nivel de Educación Básica se relacionan con el Estadio Preoperacional, el de las Operaciones Concretas y las Operaciones Formales, según los estadios de Piaget (1968); en este nivel los niños ya aprenden a través del razonamiento lógico y tiene la capacidad de plantear hipótesis, cuestionar y entender su entorno.

Por lo anterior, se considera que las Inteligencias que deberían desarrollar los docentes relacionados con la Educación General Básica son:

- Visual / Espacial

- Lingüística / Verbal

- Lógica / Matemática

- Naturalista

- Interpersonal

- Intrapersonal

Se considera que estas inteligencias pueden apoyar a los docentes que se encuentran en Educación Básica y se describe cada una de ellas a partir de las Etapas Preoperatoria, de Operaciones Concretas y de Operaciones Formales del niño. La Inteligencia Visual/ Espacial, porque el niño comprende la realidad a partir de los esquemas mentales que poseen y los estímulos visuales que requiere. La Linguística/Verbal, utiliza el lenguaje y su apropiación a partir del juego simbólico. Lógica/Matemática, porque inicia con las operaciones formales. La Naturalista donde el 
niño comprende el conocimiento científico, elabora hipótesis y razona sin tener presentes los objetos. Las inteligencias Inter e Intra personal, se considera que todo docente debe desarrollarlas para apoyar el aprendizaje integral del estudiante ya sea a partir de la reflexión o la socialización.

Finalmente, la propuesta realizada se presenta de forma sintetizada en la siguiente tabla

Tabla 3 Tipología propuesta para Educación Inicial y Básica con relación a los Estadios de Desarrollo Cognitivo de Jean Piaget

(1968) y las Inteligencias Múltiples de Gardner (1997).

\begin{tabular}{lll}
\hline Sistema Educativo & Estadios de Piaget & Inteligencias \\
\hline Educación inicial: & & Corporal / Kinestésica \\
De 0 - 5 años & Eisual / Espacial \\
(No obligatoria) & $\begin{array}{l}\text { Preopensorio Motriz y } \\
\text { Musical/Rítmica } \\
\end{array}$ & $\begin{array}{l}\text { Linguística / Verbal } \\
\text { Interpersonal }\end{array}$ \\
\hline Educación Básica: & & Visual / Espacial \\
De 5 a 14 años & Etapa Preoperatoria, & Lingüística / Verbal \\
& Operaciones Concretas y y & Natemática \\
& Operaciones Formales & Interpersonal \\
& & Intrapersonal \\
& &
\end{tabular}

Fuente: Elaboración propia con base en las teorías de Piaget (1968) y Gardner (1997)

\section{Conclusión}

Si bien es importante considerar que el desarrollo de las inteligencias es algo que debe comenzar con la propia persona, esperamos que la propuesta pueda apoyar a los profesionales de la educación Inicial y Básica a lograr el desarrollo de inteligencias que les permita diseñar ambientes de aprendizaje dirigidos a potencializar los conocimientos teniendo en cuenta la madurez cognitiva en la que el niño se encuentra.

\section{Referencias Bibliográficas}

Ardila, R. (2010). Inteligencia. ¿Qué sabemos y qué nos falta por investigar? Revista Académica Colombiana de Ciencias, 35(134), 97-103. Descargado de http://www.scielo.org.co/pdf/ racefn/v35n134/v35n134a09.pdf
Gardner, H. (1983). Frames of mind: the theory of multiple intelligences. New York: Basic Books.

Munari, A. (1994). Jean Piaget (1896-1980). Perspectivas: revista trimestral de educación comparada, XXIV(12), 315-332.

Pérez, E., y Medrano, L. (2013). Contemporary Theories of Intelligence.a Critical Review of the Literature. PSIENCIA Revista Latinoamericana de Ciencia Psicológica, 5(2), 105-118. Descargado de http://www.psiencia.org/index.php/ psiencia/article/view/113/161/ doi: 10.5872/psiencia/5.2.32

PIAGET, J. (1968). Los estadios del desarrollo intelectual del niño y del adolescente. La Habana: Editorial Revolucionaria.

Real Academia Española. (2001). Diccionario de la lengua española (22. ${ }^{\mathrm{a}}$ ed.). Descargado de http://www .rae.es/rae.html

Regader, B. (2018). ¿Qué es la inteligencia? Del CI a las inteligencias múltiples. Descargado de https://psicologiaymente.com/ inteligencia/teoria-inteligencias -multiples-gardner

Stern, W. (2018). The Psychological Methods of Testing Intelligence. CHIZINE PUBN. Descargado de https://books.google.com.ec/books ? id=qzZzSWEACAAJ

Recibido: 18 de junio de 2018

Aceptado: 29 de junio de 2018 
